\title{
VITRECTOMÍA ENZIMÁTICA POR INYECCIÓN INTRAVÍTREA DE PLASMINA AUTÓLOGA COMO TRATAMIENTO INICIAL DEL EDEMA MACULAR DIABÉTICO DIFUSO
}

\section{ENZYMATIC VITRECTOMY BY INTRAVITREAL AUTOLOGOUS PLASMIN INJECTION, AS INITIAL TREATMENT FOR DIFFUSE DIABETIC MACULAR EDEMA}

DÍAZ-LLOPIS M ${ }^{1}$, UDAONDO P², GARCÍA-DELPECH S ${ }^{2}$, CERVERA E ${ }^{1}$, SALOM D $^{2}$, QUIJADA A ${ }^{2}$

\section{RESUMEN}

Objetivo: Determinar si la inyección intravítrea de plasmina es efectiva en el tratamiento del edema macular diabético difuso (EMDD).

Diseño: Estudio piloto prospectivo, comparativo, de intervención, serie de casos.

Pacientes: Dieciocho pacientes con edema macular diabético bilateral, recibieron como primera actuación terapéutica una inyección intravítrea de plasmina en un ojo, sirviendo el ojo contralateral como control.

Tratamiento: Inyección intravítrea de $0,2 \mathrm{ml}$ de plasmina autóloga bajo anestesia tópica. La plasmina fue obtenida por un metodo simplificado.

Principales medidas: Engrosamiento macular central (EMC) medido por tomografía de coherencia óptica (OCT) y agudeza visual (escala LogMAR), al mes y los 3 meses.

Resultados: Todos los pacientes completaron el seguimiento de 3 meses. Antes de la inyección el EMC 525,22 DE 80,12 um [media \pm desviación estandard (DE)] en los ojos inyectados, versus 515,44 DE $78,13 \mu \mathrm{m}$ en los ojos control. Un mes tras la inyección el EMC era 323,72 DE 44,87 $\mu \mathrm{m}$

\section{ABSTRACT}

Purpose: To determine whether intravitreal injection of plasmin is effective in treating diffuse diabetic macular edema (DDME).

Design: A prospective, comparative, interventional case study.

Patients: Eighteen patients with bilateral DDME received, as their primary therapeutic treatment, an intravitreal injection of plasmin in one eye, with their contralateral untreated eye serving as a control. Intervention: Intravitreal $0.2 \mathrm{ml}$ of autologous plasmin injected under topical anesthesia. The plasmin was obtained by a simplified method.

Main Outcome Measures: Central macular thickness (CMT), determined by optical coherence tomography (OCT), and Best Corrected Visual Acuity (LogMAR), assessed at one and three months of follow-up.

Results: All patients completed the 3-month follow-up assessments. Before the injection, the CMT was 525.22 SD $80.12 \mu \mathrm{m}$ [mean \pm standard deviation (SD)] in the eyes to be injected, compared to $525.44 \mathrm{SD} 78.13 \mu \mathrm{m}$ in the control eyes. One

\footnotetext{
Recibido: 26/7/07. Aceptado: 29/11/07.

1 Departamento de Oftalmología. Hospital Geneneral Universitario. Unidad Docente de Oftalmología. Facultad de Medicina. Universidad de Valencia. Valencia. España.

2 Departamento de Oftalmología. Hospital Geneneral Universitario. Valencia. España.
}

Correspondencia:

Manuel Díaz-Llopis

Pl. San Agustín, 3

46002 Valencia

España

E-mail: manuel.diaz@uv.es 
en los ojos inyectados y 518,44 DE 78,54 $\mu \mathrm{m}$ en los ojos control $(\mathrm{P}<0,001$, test bilateral de Wilcoxon para muestras pareadas). A los tres meses tras la inyección el EMC era 310,55 DE 35,38 $\mu \mathrm{m}$ en los ojos inyectados y $517,66 \mathrm{DE} 80 \mu \mathrm{m}$ en los ojos control $(\mathrm{P}<0,001)$. El edema macular mejoró en todos los ojos inyectados (100\%), con una reducción al menos del $50 \%$, mientras permaneció sin cambios en el grupo control. Nueve de los 18 ojos tratados (50\%) ganaron al menos 2 líneas de visión, manteniéndose dicha mejoría durante los tres meses de seguimiento. No se observó en ningún paciente efectos secundarios.

Conclusión: La inyección intravítrea de plasmina autóloga, como tratamiento de primera elección, mejora el edema macular diabético difuso y la agudeza visual. Estudios futuros son necesarios para confirmar su eficacia y seguridad a largo plazo.

Palabras clave: Plasmina autóloga, vitreolisis farmacológica, edema macular diabético, vitrectomía enzimática. month after the injection, the CMT was $323.72 \mathrm{SD}$ $44.87 \mu \mathrm{m}$ in the injected eyes and 518.44 SD 78.54 $\mu \mathrm{m}$ in the control eyes $(\mathrm{P}<0.001$, bilateral Wilcoxon test for paired samples). Three months after the injection, the CMT was 310.55 SD $35.38 \mu \mathrm{m}$ in the injected eyes and 517.66 SD $80 \mu \mathrm{m}$ in the control eyes $(\mathrm{P}<0.001)$. Macular edema improved in all injected eyes $(100 \%)$, with a reduction of at least $50 \%$ in every treated eye, but no changes occurred in the control group. Nine of the 18 treated eyes $(50 \%)$ improved their BCVA by at least two vision lines. No adverse effects were observed in any of the patients. Conclusion: Intravitreal plasmin injection, as primary treatment, effectively reduces macular thickening due to DDME and improves visual acuity. Further studies are warranted to assess long-term efficacy and safety (Arch Soc Esp Oftalmol 2008; 83: 77-84).

Key words: Autologous plasmin, pharmacologic vitreolysis, diabetic macular edema, enzimatic vitrectomy.

\section{INTRODUCCIÓN}

El edema macular difuso diabético (EMDD) es la principal causa de emperoramiento visual en los pacientes diabéticos (1). La fotocoagulación láser en rejilla tiene una eficacia limitada (2). Diversas alternativas al tratamiento con láser han sido propuestas con variables resultados de eficacia: triamcinolona intravítrea $(3,4)$, bevacizumab intravítreo (5), inhibidores de la proteinquinasa (riboxistaurina), y análogos de la somatostatina (octeotride) $(6,7)$.

Es conocida la menor incidencia de edema macular diabético en ojos con desprendimiento posterior de vítreo (DPV) espontáneo que en aquellos con la hialoides engrosada y adherida, jugando esta tracción macular del cortex vítreo un importante papel en el aumento y cronificación del edema macular $(8,9)$. Este hecho explica que la vitrectomía, con o sin pelado de la membrana limitante interna al relajar dichas tracciones maculares ayude a la resolución del edema $(10,11)$. Pero no se debe olvidar que dicha cirugía puede tener graves complicaciones secundarias (12).

La vitreolisis farmacológica por inyección de plasmina autóloga ha sido propuesta por diversos autores $(13,14)$. La plasmina, es una proteasa implicada en la fibrinolisis, con acción enzimática sobre la laminina y la fibronectina, que están localizadas entre el cortex vítreo posterior y la membrana limitante interna de la retina, y se consideran las principales moléculas implicadas como responsables de la firme adhesión entre ambas superficies $(15,16)$. Diversos estudios han comunicado la eficacia de la inyección de plasmina, inmediatamente previo a la vitrectomía, en casos de retinopatía diabética y agujeros maculares, simplificando la cirugía al facilitar el despegamiento quirúrgico de las membranas vitreoretinales (17-19).

El objetivo del presente estudio piloto, prospectivo y controlado, sobre una serie de pacientes diabéticos con edema macular difuso bilateral, es establecer la eficacia y seguridad de la inyección autóloga de plasmina — sin asociar vitrectomía-, como única opción e inicial terapéutica en el tratamiento de dicho EMDD, evaluando dicha eficacia en función de los cambios que se produzcan tanto en la agudeza visual como en la tomografía de coherencia óptica.

\section{SUJETOS, MATERIAL Y MÉTODO}

Este estudio es un estudio piloto prospectivo y de intervención terapéutica. Los pacientes fueron 
reclutados entre septiembre 2006 y febrero 2007 en la Unidad de Diabetes del Servicio de Oftalmología, contando con la aprobación del Comité de Investigación del Hospital. Treinta y seis ojos de dieciocho pacientes consecutivos fueron incluidos en el estudio, utilizándose un ojo de control y el contralateral para tratamiento con plasmina intravítrea.

\section{Pacientes: criterios de inclusión y exclusión}

\section{Criterios de inclusión}

Sólo se incluyeron pacientes diabéticos que tuvieran edema macular difuso bilateral. El edema macular difuso débía cumplir en ambos ojos los siguientes criterios: a) Engrosamiento macular confirmable por biomicroscopía y lente de $90 \mathrm{D}$ b) Escapes generalizados por el área macular en la angiografía fluoresceínica c) Ausencia de fotocoagulación láser previa retiniana (macular o de la periferia) d) Confirmación por tomografía de coherencia óptica (Stratus OCT-3, Zeiss) de un engrosamiento macular mayor de $360 \mu \mathrm{m}$ (normal $=/<200$ $\mu \mathrm{m})$.

\section{Criterios de exclusión}

1. Presión arterial no controlada, sistólica y diastólica mayor de 150 y $90 \mathrm{mmHg}$ respectivamente.

2. Niveles de glucemia no estables. Hemoglobina glicosilada (HB A1c) mayor de 9,5\%.

3. Insuficiencia renal.

4. Historia ocular de hipertensión ocular, glaucoma.

5. Signos de isquemia macular o de la periferia retiniana en la angiografía fluoresceínica.

6. Cirugía ocular durante los seis meses previos.

7. Desprendimiento de vítreo posterior previo, diagnosticado por biomicroscopía y lente de +90D y/o tomografía de coherencia óptica.

Todos los pacientes firmaron consentimiento informado, explicándoles claramente el objetivo del estudio y los potenciales riesgos (desprendimiento de retina, endoftalmitis, hemorragia vítrea, traumatismo del cristalino, etc.) y la posibilidad de requerir tratamientos adicionales posteriores para su retinopatía diabética (láser, intravítreas de fármacos anti-VEGF o triamcinolona).

\section{Examen preoperatorio}

Todos los pacientes recibieron una exploración oftalmológica completa antes de la inyección de plasmina, incluyendo exploración a la lámpara hendidura, tonometría de aplanación, oftalmoscopía indirecta, escaner macular por tomografía de coherencia óptica, fotografía de fondo del áera macular y angiografía fluoresceínica. La tomografía de coherencia y la biomicroscopía descartaron desprendimiento del vítreo y confirmaron su adherencia del cortex vítreo en el área macular en todos los casos.

\section{Preparación de la plasmina autóloga}

La plasmina fue preparada en el quirófano inmediatamente antes de la inyección, como ha sido descrita previamente (20). Una muestra de sangre del propio paciente se extrajo de una vena periférica. Una vez centrifugada la sangre a 4,000 rpm durante 15 minutos, el plasma fue transferido a un vial con estreptoquinasa (Streptase ${ }^{\circledR}$, ZLB BEHRIG Laboratories), que había sido incubado previamente durante 15 minutos a $37^{\circ} \mathrm{C}$. La estreptoquinasa disuelta fue mezclada con el plasma agitando el vial vigorosamente durante 5 minutos. La solución volvió a ser incubada durante 15 minutos más a $37^{\circ} \mathrm{C}$. Finalmente, la solución fue esterilizada a través de un filtro milipore de $0,22 \mathrm{~mm}$, y ya estaba lista para ser inyectada.

\section{Elección del ojo y Técnica de Inyección}

Los ojos fueron asignados de manera aleatoria al grupo con plasmina o al grupo control. Antes de la inyección, se realizó anestesia tópica con colirio de tetracaína al $1 \%$ al menos tres veces y lavado conjuntival con solución de povidona. Paracentesis de cámara anterior con aguja de $25 \mathrm{G}$. A continuación se realizó inyección intravítrea de la solución de plasmina autóloga con aguja de $30 \mathrm{G}$, a 3,5 ó $4 \mathrm{~mm}$ del limbo dependiendo de que el paciente fuera pseudofáquico o fáquico respectivamente. Aplicación de hemosteta en el punto de inyección para evitar el reflujo. Colirio de ciprofloxacino y dexametasona 4 veces día durante los cinco días postoperatorios. 


\section{Principales Medidas}

Las principales medidas fueron los cambios en la agudeza visual y en el engrosamiento macular central (EMC). Para evitar sesgos y diferencias interobservador, todas las agudezas visuales (optotipos ETDRS) y las mediciones del engrosamiento macular en la tomografía de coherencia (OCT-3, ZeissHumphrey, Dublin, CA, USA) fueron medidas por un único explorador que desconocía los datos clínicos de los pacientes (P.U.). Con fines estadísticos la mejor agudeza visual corregida medida (MAVC) fue convertida en equivalente $\log$ MAR del mínimo ángulo de resolución. Las mediciones se realizaron el día previo, a las 4 y 12 semanas de la inyección. Se consideró el valor medio del espesor central retiniano normal de $200 \mu \mathrm{m}$.

\section{Estudio estadístico}

Sólo un ojo por paciente fue tratado. Todos los datos fueron procesados usando el programa SPSS 13.0 para Windows (SPSS for Windows, SPSS Inc, Chicago, USA). Para el estudio estadístico de datos pareados, cada ojo tratado fue pareado con el ojo no-tratado del mismo paciente. El estudio estadístico fue realizado usando el test bilateral de Wilcoxon para datos pareados, considerándose $\mathrm{P}<0,05$ con significación estadística.

\section{RESULTADOS}

Treinta y seis ojos fueron incluidos en el estudio procedentes de un total de dieciocho pacientes, siendo utilizado siempre uno de los ojos como control- únicamente observación- y el contralateral para inyección intraocular de plasmina. La edad media de los pacientes era de 65 años (rango 5683). Doce eran mujeres y seis hombres. Trece eran pseudofáquicos y cinco fáquicos. Se inyectó plasmina en nueve ojos derechos y nueve ojos izquierdos. Todos los pacientes tenían retinopatía diabética no-proliferante.

\section{Agudeza visual (tabla I)}

No existieron diferencias estadísticamente significativas entre las medias de las agudezas visuales
Tabla I. Mejor agudeza visual corregida (MAVC) de los ojos tratados con inyección de plasmina autóloga intravítrea (PAI) antes, al mes y a los tres meses después del tratamiento (valores convertidos a escala LogMar)

\begin{tabular}{|c|c|c|c|}
\hline \multirow[t]{2}{*}{$\begin{array}{l}\text { N. } \\
\text { Paciente }\end{array}$} & \multicolumn{3}{|c|}{$\begin{array}{l}\text { Agudeza visual ojos tratados } \\
\text { con plasmina autóloga }\end{array}$} \\
\hline & Pre-tratamiento & $1 \mathrm{mes}$ & 3 meses \\
\hline 1 & 0,3 & 0,18 & 0,18 \\
\hline 2 & 0,48 & 0,3 & 0,3 \\
\hline 3 & 1,0 & 1,0 & 1,0 \\
\hline 4 & 0,9 & 0,48 & 0,48 \\
\hline 5 & 0,78 & 0,6 & 0,48 \\
\hline 6 & 0,48 & 0,3 & 0,3 \\
\hline 7 & 0,6 & 0,48 & 0,48 \\
\hline 8 & 0,9 & 0,6 & 0,6 \\
\hline 9 & 1,0 & 0,9 & 0,9 \\
\hline 10 & 0,9 & 0,78 & 0,6 \\
\hline 11 & 0,6 & 0,48 & 0,48 \\
\hline 12 & 0,9 & 0,6 & 0,48 \\
\hline 13 & 1,0 & 1,0 & 1,0 \\
\hline 14 & 0,3 & 0,18 & 0,18 \\
\hline 15 & 0,48 & 0,48 & 0,3 \\
\hline 16 & 0,6 & 0,48 & 0,48 \\
\hline 17 & 0,9 & 0,6 & 0,48 \\
\hline 18 & 1,0 & 0,9 & 0,9 \\
\hline
\end{tabular}

preoperatorias del grupo control $(1,04$, datos no mostrados) y la del grupo tratado con plasmina autóloga intravítrea (PAI) $(1,03)(\mathrm{P}>0,05)$. No hubo cambios significativos en la MAVC del grupo control a los 3 meses del seguimiento (MAVC media 1,03), mientras la MAVC media del grupo tratado con plasmina mejoró a 0,53 . Es decir, la mejoría media de la MAVC fue de 0,50 unidades logarítmicas del mínimo ángulo de resolución en el ojo tratado con plasmina y sólo de 0,01 en el grupo control. Esta diferencia es clínica y estadísticamente significativa $(\mathrm{P}<0,001)$.

La AV mejoró en dieciséis de los dieciocho ojos tratados: en siete ojos una línea de visión, en cuatro ojos dos líneas, en dos ojos tres líneas y en tres ojos cuatro líneas.

\section{Espesor macular central (tabla II)}

El espesor macular central medio (EMC) del ojo a tratar $(525,22 \mathrm{DE} 80,12 \mu \mathrm{m})$ con plasmina fué discretamente mayor que el ojo control $(515,44 \mathrm{DE}$ $78,13 \mu \mathrm{m})$, pero sin significación estadística ( $\mathrm{p}>$ $0,05)$. Un mes tras la inyección de plasmina el espesor macular central medio era en el ojo tratado y en el ojo control 322,72 DE $44,87 \mu \mathrm{m}$ y $518,44 \mathrm{DE}$ 
Tabla II. Espesor macular central en los ojos tratados con plasmina autóloga intravítrea (PAI) y en los ojos control medido por OCT pre-tratamiento, al mes y a los tres meses

\begin{tabular}{lcccccc}
\hline N. ${ }^{\circ}$ Paciente & & Ojos Tratados PAI & & Ojos Control \\
1 mes & 3 meses & Inicio & mes & 3 meses \\
\hline 1 & Pre IPA & 410 & 367 & 561 & 567 & 572 \\
2 & 569 & 305 & 311 & 605 & 619 & 614 \\
3 & 624 & 373 & 314 & 446 & 441 & 459 \\
4 & 451 & 329 & 335 & 551 & 541 & 560 \\
5 & 555 & 321 & 305 & 526 & 532 & 521 \\
6 & 532 & 297 & 276 & 456 & 461 & 431 \\
7 & 467 & 342 & 346 & 571 & 590 & 582 \\
8 & 579 & 286 & 272 & 433 & 442 & 437 \\
9 & 446 & 317 & 291 & 507 & 515 & 502 \\
10 & 512 & 308 & 296 & 497 & 525 & 516 \\
11 & 509 & 271 & 293 & 478 & 462 & 480 \\
12 & 481 & 384 & 375 & 620 & 635 & 622 \\
13 & 632 & 403 & 374 & 684 & 653 & 662 \\
14 & 303 & 247 & 254 & 357 & 349 & 338 \\
15 & 405 & 274 & 281 & 416 & 426 & 423 \\
16 & 546 & 326 & 315 & 529 & 536 & 549 \\
17 & 490 & 279 & 268 & 479 & 471 & 481 \\
18 & 573 & 337 & 317 & 564 & 572 & 569 \\
Media+/-DE & $525,22 \pm 80,12$ & $322,72 \pm 44,87$ & $310,55 \pm 35,58$ & $515,44 \pm 78,13$ & $518,72 \pm 78,54$ & $517,66 \pm 80$ \\
\hline \hline
\end{tabular}

DE: desviación estándar; PAI: inyección de plasmina autóloga; Nota: los valores de OCT se expresan en $\mu$.

$78,13 \mu \mathrm{m}$, respectivamente. Esta diferencia estadísticamente significativa $(\mathrm{P}<0,001$, tests de Wilcoxon bilateral para datos pareados) entre ambos grupos se mantenía a los 3 meses (310,55 DE 15,58 $\mu \mathrm{m}$ -plasmina- versus 517,66 DE $80 \mu \mathrm{m}$-control-). Mientras no hubo cambios en el grupo control significativos durante el seguimiento, el espesor macular central se redujo en el grupo tratado en todos los casos: más del $70 \%$ en cuatro ojos, del 60 al $70 \%$ en once ojos y más del $50 \%$ en los restantes tres ojos, considerando un espesor macular central normal de $\leq 200 \mu \mathrm{m}$.

\section{Efectos secundarios}

No se observaron efectos secundarios durante el seguimiento.

\section{DISCUSIÓN}

Los resultados de este estudio prospectivo y controlado creemos han demostrado la eficacia de la inyección intravítrea de plasmina en mejorar la agudeza visual y reducir el engrosamiento retiniano en los pacientes con edema macular difuso diabético (EMDD). Hasta la actualidad la inyección intraví- trea de plasmina se había utilizado siempre inmediatamente antes de la vitrectomía para facilitar el manejo intraoperatorio $(17,19,21)$, pero este estudio ha demostrado la eficacia de la inyección aislada de plasmina como único tratamiento. La separación farmacológica del cortex posterior vítreo de la retina sólo con la inyección intravítrea de plasmina, sin asociar vitrectomía, que creemos debería llamarse «vitrectomía enzimática», abre la posibilidad terapéutica de tratamiento no sólo del edema macular diabético, sino de otros edemas maculares (oclusiones venosas, etc.) y otras patologías donde la tracción vitreoretinal esté claramente implicada en su patogenie (síndrome de tracción macular miópica, etc), sin los riesgos yatrogénicos asociados de la cirugía vítrea (desgarros y desprendimientos de retina, hemorragia vítrea etc).

Aunque puede no existir una correlación directa entre mejoría de la agudeza visual y la reducción del edema macular diabético tras la inyección de plasmina, la reducción del edema fue mayor del $50 \%$ en todos los ojos tratados (100\%), con una mejoría de dos o más líneas de visión en el 50\% de los casos. Debemos recordar que la inyección de plasmina se utilizó como primera opción terapéutica del edema macular difuso diabético antes que intentar su tratamiento con láser, triamcinolona, pegaptanib, ranibizumab o bevacizumab intravítreo. 
Esta mejoría visual es percibida por el paciente muy precozmente (2-3 días), manteniéndose estable desde el primero hasta el tercer mes de seguimiento. Este estudio es piloto y por tanto sus resultados no generalizables. Estudios con mayor número de casos y seguimientos a largo plazo son necesarios para establecer su eficacia como monoterapia o la necesidad de asociar el resto de tratamientos anteriormente descritos para consolidar su eficacia.

Por otro lado, en nuestro estudio hemos observado un desprendimiento del vítreo posterior (DVP) completo del área macular en todos nuestros pacientes (fig. 1), documentado clínicamente por biomicroscopía o por tomografía de coherencia
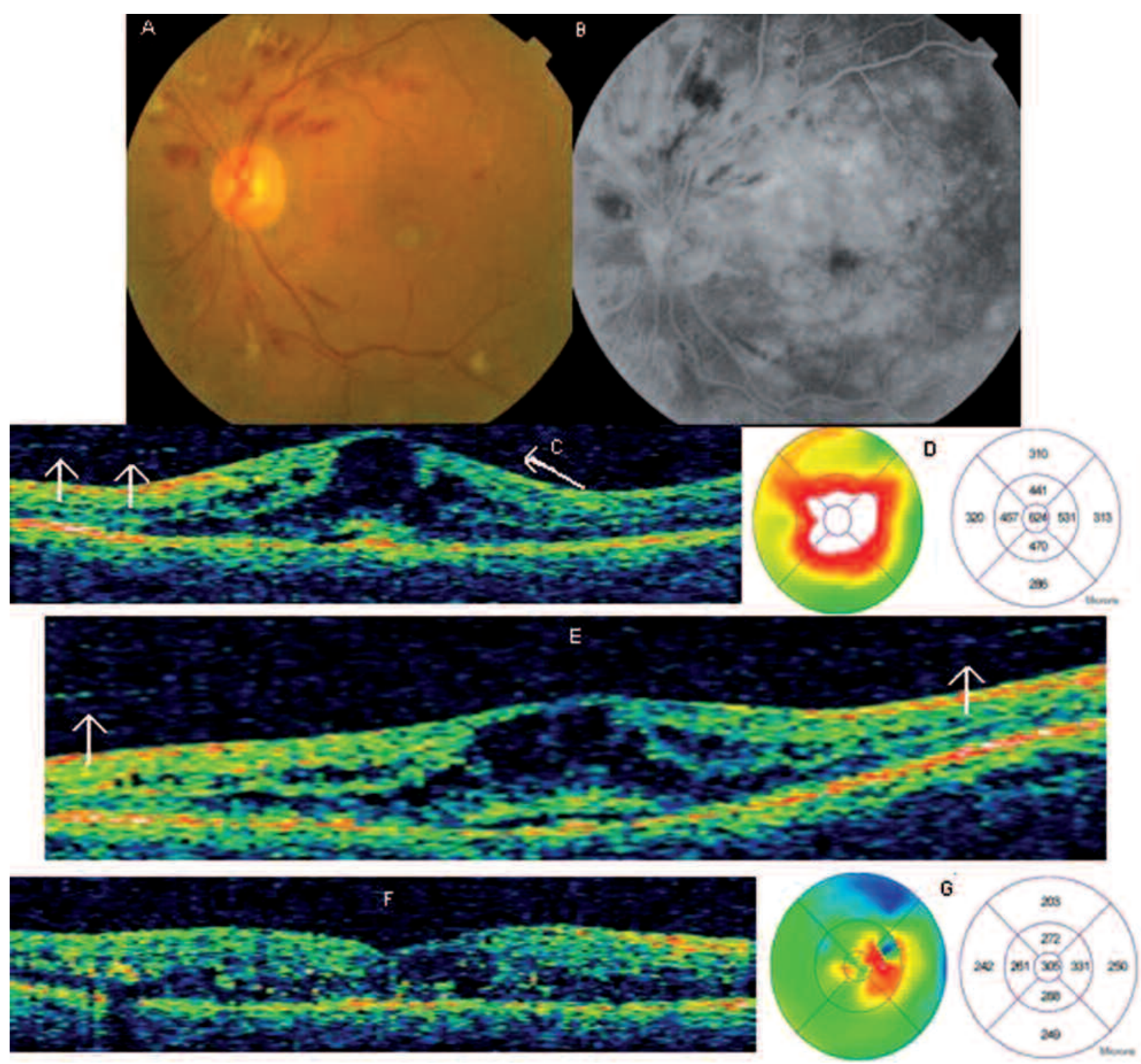

Fig. 1: A,B: Imagen de fondo de ojo y angiografía correspondiente a un paciente diabético con un gran edema macular difuso-paciente $n^{\circ} 2$-. $C, D, E, F, G$ : Serie de imágenes de tomografía de coherencia óptica (OCT) que corresponde al mismo paciente antes $(C, D)$, una semana $(E)$ y un mes después $(F, G)$ del tratamiento con plasmina autóloga; las flechas muestran con detalle la hialoides posterior traccionando antes de la inyección y como se va desprendiendo tras la inyección de plasmina, mejorando simultáneamente de manera notable el edema macular, reduciéndose el engrosamiento macular desde 624 a 305 micras. 
óptica, con un significativo descenso del engrosamiento macular retiniano - edema macular anatómico- en todos los casos (100\%). Dicha resolución del edema macular se mantuvo estable durante los tres meses de seguimiento del presente estudio, pero son imprescindibles estudios a más largo plazo para establecer la existencia de recidivas y/o la necesidad de asociar otras ópciones terapéuticas (láser, antiVEGF o triamcinolona intravítrea).

La eficacia y toxicidad de la plasmina autóloga es dosis-dependiente. La inyección intravítrea de 0,4 UI de plasmina ha demostrado en estudios previos ser suficiente para separar el cortex vítreo posterior de la limitante interna, sin toxicidad conocida hasta concentraciones de 3-4 UI $(4,17,22,24,25,27)$.

La cantidad de plasmina autóloga obtenida e inyectada por el método empleado en este estudio es de media de 0,26 UI (20), pero ha demostrado ser suficiente para ser eficaz. Por otro lado, Gandorfer et al (13) encontraron una correlación directa entre tiempo de exposición de la plasmina y grado de separación vítreo-retiniana, partiendo del principio que la plasmina autóloga residual en los estudios previos ha sido siempre evacuada del vítreo en la maniobra quirúrgica inmediatamente posterior a la inyección realizada. El tiempo de exposición de la superficie vitreo-retinal a la plasmina autóloga ha demostrado en el presente estudio ser suficiente y sin toxicidad. Revisado PubMed es el tiempo más largo de exposición investigado en la literatura $(25,26)$. Tampoco se ha observado en ningún caso complicación alguna como endoftalmitis, hemorragia vítrea, uveítis, desprendimiento de retina, aumento de la presión intraocular o progresión de catarata.

Uno de los principales problemas que limitaban el uso de plasmina autóloga ha sido siempre su sofisticado sistema de preparación, largo, costoso y complejo, requiriendo equipamientos especializados sólo accesibles en determinadas unidades hematológicas. La ventaja de la técnica simplificada de preparación de plasmina autóloga es la posibilidad de ser preparada en el propio quirófano de Oftalmología unos minutos antes de su uso, y de manera rápida, fácil y relativamente económica (20).

En conclusión, la vitrectomía enzimática realizada únicamente con la inyección de plasmina autóloga ha demostrado ser eficaz y segura en reducir el edema macular difuso diabético y mejorar la agudeza visual, al menos a corto plazo, y parece ser una buena, primera e inicial opción terapéutica en estos pacientes antes de ensayar otras opciones terapéuticas (láser, triamcinolona intravítrea, etc.). Futuros estudios deberán matizar la dosis de plasmina más idónea, la posibilidad de recurrencias a largo plazo y la conveniencia de los tratamientos complementarios asociados que posteriormente puedan ser necesarios.

\section{BIBLIOGRAFÍA}

1. Klein R, Klein BE, Moss SE. Visual impairment in diabetes. Ophthalmology 1984; 91: 1-9.

2. Photocoagulation for diabetic macular edema. Early Treatment Diabetic Retinopathy Study report number 1. Early Treatment Diabetic Retinopathy Study Research Group. Arch Ophthalmol 1985; 103: 1796-1806.

3. Massin P, Audren F, Haouchine B, Erginay A, Bergmann $J F$, Benosman $R$, et al. Intravitreal triamcinolone acetonide for diabetic diffuse macular edema: preliminary results of a prospective controlled trial. Ophthalmology 2004; 111: 218-225.

4. Martidis A, Duker JS, Greenberg PB, Rogers AH, Puliafito $C A$, Reichel $E$, et al. Intravitreal triamcinolone for refractory diabetic macular edema. Ophthalmology 2002; 109: 920-927.

5. Haritoglou C, Kook D, Neubauer A, Wolf A, Priglinger S, Strauss $R$, et al. Intravitreal bevacizumab (Avastin) therapy for persistent diffuse diabetic macular edema. Retina 2006; 26: 999-1005.

6. Comer GM, Ciulla TA. Pharmacotherapy for diabetic retinopathy. Curr Opin Ophthalmol 2004; 15: 508-518.

7. Lang GE. Pharmacological treatment of diabetic retinopathy. Ophthalmologica 2007; 221: 112-117.

8. Schepens CL, Avila MP, Jalkh AE,Trempe CL. Role of the vitreous in cystoid macular edema. Surv Ophthalmol 1984; 28: 499-504.

9. Ikeda T, Sato K, Katano T, Hayashi Y. Attached posterior hyaloid membrane and the pathogenesis of honeycombed cystoid macular edema in patients with diabetes. Am J Ophthalmol 1999; 127: 478-479.

10. Tachi N, Ogino N. Vitrectomy for diffuse macular edema in cases of diabetic retinopathy. Am J Ophthalmol 1996; 122: 258-260.

11. Yamamoto T, Akabane N, Takeuchi S. Vitrectomy for diabetic macular edema: The role of posterior vitreous detachment and epimacular membrane. Am J Ophthalmol 2001; 132: 369-377.

12. Yamamoto T, Hitani K, Tsukahara I, Yamamoto S, Kawasaki $R$, Yamashita $H$, et al. Early postoperative retinal thickness changes and complications after vitrectomy for diabetic macular edema. Am J Ophthalmol 2003; 135: 1419.

13. Gandofer A, Putz E, Welge-Lüssen U, Grüterich M, Ulbig $M$, Kampik A. Ultrastructure of the vitreoretinal interface following plasmin assisted vitrectomy. Br J Ophthalmol 2001; 85: 6-10.

14. Asami T, Terasaki H, Kachi S, Nakamura M, Yamamura $K$, Nabeshima $T$, et al. Ultrastructure of internal limiting membrane removed during plasmin-assisted vitrectomy 
from eyes with diabetic macular edema. Ophthalmology 2004; 111: 231-237.

15. Li X, Shi X, Fan J. Posterior vitreous detachment with plasmin in the isolated human eye. Graefes Arch Clin Exp Ophthalmol 2002; 240: 56-62.

16. Tanaka M, Qui H. Pharmacological vitrectomy. Semin Ophthalmol 2000; 15: 51-61.

17. Azzolini C, D'Angelo A, Maestranzi G, Codenotti M, Della Valle P, Prati M, et al. Intrasurgical plasmin enzyme in diabetic macular edema. Am J Ophthalmol 2004; 138 : 560-566.

18. Sakuma T, Takana M, Inoue M, Mizota A, Souri M, Ichinose A. Efficacy of autologous plasmin for idiopathic macular hole surgery. Eur J Ophthalmol 2005; 15: 787794.

19. Williams JG, Trese MT, Williams GA, Hartzer MK. Autologous plasmin enzyme in the surgical management of diabetic retinopathy. Ophthalmology 2001; 108: 1902-1905.

20. Rizzo S, Pellegrini G, Benocci F, Belting C, Baicchi $M$, Vispi M. Autologous plasmin for pharmacologic vitreolysis prepared 1 hour before surgery. Retina 2006; 26: 792-796.

21. Margherio AR, Margherio RR, Hartzer M, Trese MT, Williams GA, Ferrone PJ. Plasmin enzyme-assisted vitrectomy in traumatic pediatric macular holes. Ophthalmology 1998; 105: 1617-1620.

22. Gandorfer A, Rohleder M, Sethi C, Eckle D, Welge-Lüssen $U$, Kampik A, et al. Posterior vitreous detachment induced by microplasmin. Invest Ophthalmol Vis Sci 2004; 45: 641-647.

23. Massin P, Vicaut E, Haouchine B, Erginay A, Paques M, Gaudric A. Reproducibility of retinal mapping using optical coherence tomography. Arch Ophthalmol 2001; 119: 1135-1142.

24. Wang F, Wang Z, Sun X, Wang F, Xu X, Zhang X. Safety and efficacy of dispase and plasmin in pharmacologic vitreolysis. Invest Ophthalmol Vis Sci 2004; 45: 32863290.

25. Gandorfer A. Pharmacologic vitreolysis. Dev Ophthalmol 2007; 39: 149-156.

26. Trese MT. Enzymatic vitreous surgery. Semin Ophthalmol 2000; 15: 116-121.

27. Hermel M, Mahgoub M, Youssef T, Azrak MI, Raza H, Alldredge $C$, et al. Safety profile of the intravitreal streptokinase-plasmin complex as an adjunct to vitrectomy in the rabbit. Graefes Arch Clin Exp Ophthalmol 2006; 244 : 996-1002. 